\title{
Synthesis and Characterization of Starch-Graft-Acrylamide Hydrogel for Oral Drug Delivery
}

\author{
*H. Musa, Y. Musa and M. Suleiman \\ Department of Pure and Industrial Chemistry, Bayero University, P.M.B. 3011, Kano Nigeria \\ [Corresponding Author: E-mail: hmusa.chm@buk.edu.ng; ? +2348032552885]
}

\section{ABSTRACT}

In this research, starch was extracted from fresh sweet potato and was used to prepare starch-gacrylamide hydrogel using free radical polymerization method with potassium per sulphate and N'NMethylene bisacrylamide as initiator and cross-linker, respectively. The swelling capacity and $\mathrm{pH}$ sensitivity of the synthesized hydrogel were investigated in solutions of various $\mathrm{pH}(1-12)$. The drug loading and release experiment was also carried out using promethazine (PMZ) as the model drug at $25^{\circ} \mathrm{C}$ and $37{ }^{\circ} \mathrm{C}$, respectively while the release study was carried out in an enzyme-free simulated gastric intestinal fluid (SGF) and simulated intestinal fluid (SIF). The result showed a 905\% swelling at pH 11, suggesting increased swelling capacity at higher $\mathrm{pH}$ values. Drug loading result indicated $99 \%$ of the drug was entrapped by the hydrogel as confirmed by UV-visible spectrophotometry. SIF and SGF Simulation indicated a $24 \%$ and $9 \%$ drug release for the first ten hours. At the end of 48 hours the release was $96 \%$ and $89 \%$, respectively indicating the hydrogel released more promethazine in SIF than in SGF. The results obtained in this work suggest that starch-graft-acrylamide hydrogel is a potential vehicle for oral drug delivery.

Keywords: Starch, Acrylamide, Hydrogel, Drug delivery.

\section{INTRODUCTION}

An area of research that received great attention and progressed greatly in the past few decades is the utilization of hydrogels in the biomedical applications as drug carriers (Peppas, 2000). The most interesting class of polymers in the application of drug delivery system using solid matrices is the hydrogel (Sadeghi, 2011). Initially, drugs could only be administered in a limited manner due to limitations of transportation route through body harmful environment. Thus, limited mobility and other problems associated with old method have made the drug delivery tedious and reduced the efficacy of the administered drugs (Azman et al., 2015). Great development has been achieved with the invention of biomaterial carriers, which could be encapsulated with drug, allowing the drug to safely reach the targeted body site without any harm. These carriers allow the drug to simply access the body sites which were previously inaccessible (Peppas, 2000).

Hydrogels are three-dimensional cross-linked polymer matrices that are capable of absorbing large amount of water or biological fluids without being dissolve (Esra et al., 2007). The hydrogels ability to absorb water is due to the presence of hydrophilic functional groups attached to the polymeric backbone, while their resistance to dissolution is due the crosslinks between the network chains (Ahmed, 2015). Since the pioneering work of Wichterle and Lim in 1960 on crosslinked hydrogels (Kopecek, 2007) and because of their hydrophilic character, biocompatibility and biodegradability hydrogels has been an area of great interest to biomaterial scientists for many years (Kono and Teshirogi, 2015). Hydrogels have been actively studied, particularly those experiencing reversible volume changes in response to external stimulus, such as $\mathrm{pH}$, temperature and ionic concentration. These "smart" hydrogels have found applications in biomedicine and biotechnology including soft contact lenses (Soleimani and Sadeghi, 2012), immobilization of enzymes and proteins (Niamlang et al., 2013), antibodies and antigens and matrices for drug delivery systems (Park et al., 2013). The ability of these hydrogels to respond to their environment increase drug loading and provide protection from environmental conditions such as those found in the gastrointestinal tract (Michel et al., 2011). 
In this regard, stimuli responsive hydrogels can be useful for the design of a specific site drug delivery system; for instance, colon-targeted drug delivery systems (Usman et al., 2015). Another important advantage of these hydrogels is that the active ingredient remains on the organ or tissue for longer times than when taken using conventional method (Shanta and Harding, 2002). Drug delivery systems through hydrogel enhances therapeutic efficacy, minimize side effects, and improve patient compliance (Muhammad et al., 2016). The hydrogels simply work in response to an environmental stimulus such as temperature, ion concentration, $\mathrm{pH}$ and electric field and release its contents in a fashionable manner over a specified period of time. One of the properties of hydrogel is its unique ability to swell thousand times its dried weight (Jamingan et al., 2015). This research is aimed at synthesizing biodegradable and biocompatible hydrogels for oral drug delivery that could overcome some of the persistent problems and limitations associated with the conventional drug administration.

\section{MATERIALS AND METHOD}

Native sweet potato tuber was purchased from Dawanau market, Kano state. Acrylamide $(98 \%)$ and ethanol $(96 \%)$ were purchased from LobaChemie, N'N-Methylene bisacrylamide
(97\%) from Sigma Aldrich, USA. Potassium persulphate (98\%) from Qualichem,.All other reagents are of analytical grade and were used as received. All glasswares were cleaned thoroughly, rinsed with deionised water and dried in an oven at $100{ }^{\circ} \mathrm{C}$ overnight.

\section{Synthesis of Starch-g-Acrylamide Hydrogel Film}

Starch acrylamide hydrogel was synthesized using a modified procedure as described by Soleimani and Sadeghi (2012). Potato starch (4 g) was dissolved in $100 \mathrm{ml}$ of distilled water in a three-necked round bottom flask and placed in a pre-heated water bath with constant stirring in nitrogen gas atmosphere. After 15 minutes, acrylamide $(8 \mathrm{~g})$ was dissolved in $50 \mathrm{ml}$ of distilled water and added into the three-necked round bottom flask containing the starch solution under vigorous stirring $(200 \mathrm{rpm})$ at $55^{\circ} \mathrm{C}$. After 15 minutes, the mixture became homogeneous and potassium persulphate $(0.1$ $\mathrm{M})$ and N'N-methylene bisacrylamide (0.2 M) as an initiator and crosslinker, respectively were added. The Synthesis was allowed to proceed for 2 hours at $65-75^{\circ} \mathrm{C}$, after which the synthesized hydrogel was washed with ethanol followed by water and dried to a constant weight in an oven at $60^{\circ} \mathrm{C}$.

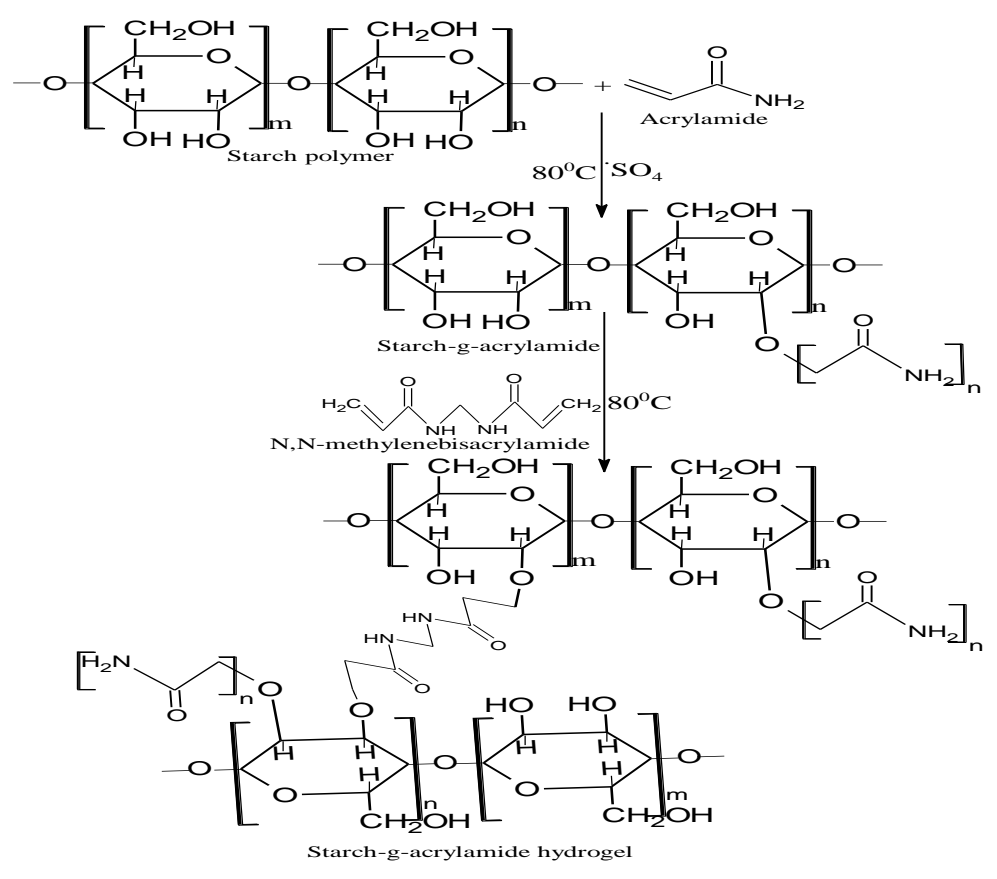

Scheme 1: Synthesis of starch-g-acrylamide Hydrogel 


\section{CHARACTERIZATION}

Fourier Transform Infrared Spectroscopy (FTIR)

Aliquot $(0.2 \mathrm{~g})$ of the prepared hydrogel was ground to powder and its FTIR spectrum was obtained using CARRY 4000 FTIR AGILENT spectrometer within the range of $4000-600$ $\mathrm{cm}^{-1}$.

\section{Scanning Electron Microscopy (SEM)}

Powdered form of the synthesized hydrogel was taken to observe its surface morphology before and after drug loading by scanning at different magnifications using Scanning Electron Microscope Phenom Pro X Model.

\section{Swelling Studies}

The swelling capacity of hydrogel was evaluated according to the method of Ahmed (2015). The swelling behaviour of the hydrogel film was carried out in solutions of various $\mathrm{pH}$ (1-12) and was determined gravimetrically. A solution of $\mathrm{pH} 1-12$ was prepared and a known weight of the dried hydrogel films was immersed in each solution and left until an equilibrium was reached after which the swollen hydrogel films were removed and weighed, while weighing the swollen hydrogel films the water on the films surface were wiped with filter paper so as to ensure only the entrapped liquid by the hydrogel films was weighed. The swelling percentage of the hydrogels was calculated according to the following equation:

$$
\mathrm{S}(\%)=\frac{W_{s}-W d}{W_{s}} x 100
$$

Where $W_{s}$ and $W_{d}$ are the weights of the swollen and dried hydrogel films respectively.

\section{Preparation of Promethazine Solution}

A promethazine solution of $1 \mathrm{mg} / \mathrm{ml}$ was prepared by taking $1 \mathrm{~g}$ of the drug, dissolved in distilled water, and made up to the mark of $1 \mathrm{~L}$ capacity volumetric flask.

\section{Drug Loading}

The loading of promethazine drug was done by weighing a known weight $(2 \mathrm{~g})$ of the hydrogel and immersed into the solution containers for adsorption to take place for 24 hours (equilibrium swelling) after which the hydrogel was removed from the solution and dried at room temperature for 48 hours. The residual concentration of the solution was measured to determine the percentage of drug entrapment in the hydrogel using Perkin Elmer UV- Visible Spectrometer at a wavelength of $254 \mathrm{~nm}$. The percentage drug entrapment was calculated using the following equation.

$$
\% \mathrm{~A}=\frac{\mathrm{Ci}-\mathrm{Cf}}{\mathrm{Ci}} \times 100
$$

Where $C_{i}$ and $C_{f}$ are the initial and final concentration of the drug respectively (Muhammad et al., 2016)

\section{In Vitro Release of Promethazine Drug}

The in vitro release of the entrapped promethazine drug was carried out by placing the hydrogel pellet loaded with the promethazine drug in an enzyme-free simulated gastric fluid (SGF) and simulated intestinal fluid (SIF) at $37^{\circ} \mathrm{C}$. The release study was carried out in INNOVA 4000 INCUBATOR SHAKER with reciprocating motion of (100 rpm). After each periodic interval, the release medium was replaced with a fresh simulated gastric fluid and simulated intestinal fluid.

\section{RESULTS AND DISCUSSION}

Figure 1 shows the FTIR spectrum of the synthesized hydrogel with the key functional groups highlighted.

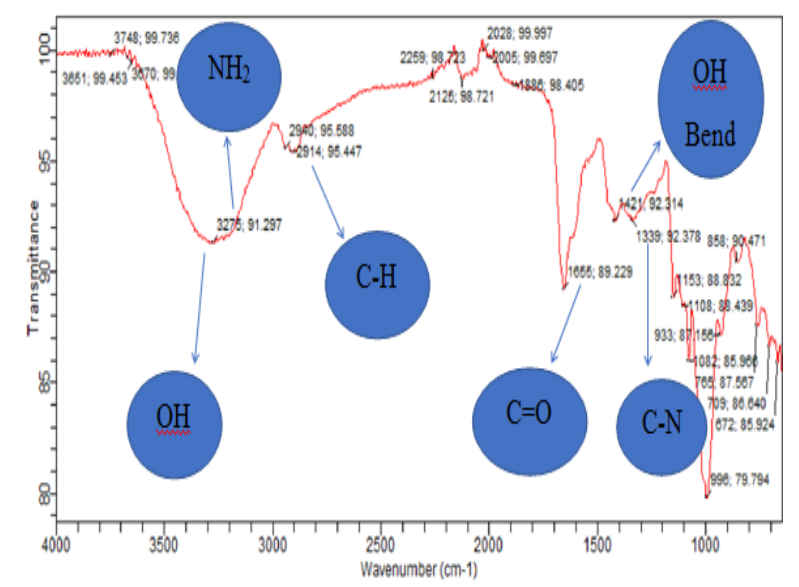

Figure 1: FTIR Spectrum of Starch-gacrylamide Hydrogel

The IR spectrum showed the absorption bands at $3275 \mathrm{~cm}^{-1}$ for (N-H stretching), $1655 \mathrm{~cm}^{-1}$ for ( $\mathrm{C}=\mathrm{O}$ of amide stretching), $2936 \mathrm{~cm}^{-1}$ for $(\mathrm{C}-\mathrm{H})$ 
and broad band at $3650 \mathrm{~cm}^{-1}$ for $(\mathrm{OH}$ stretching). There is also a band at $1339 \mathrm{~cm}^{-1}$ for (-C-N-) group of the crosslinking agent (N'Nmethylene bisacrylamide) and a presence of another band at $1421 \mathrm{~cm}^{-1}$ for $(\mathrm{OH})$ bending. Since the polymeric hydrogel has been extracted to remove the soluble content, the presence of these key functional groups in the spectrum has indicated the successful formation of starch-g-acrylamide hydrogel.

Figure 2 shows the images obtained from scanning electron microscopy of the prepared hydrogel before and after drug loading. The first image (Figure 2a) shows the surface of the hydrogel before promethazine loading while Figure $2 b$ is the surface of the hydrogel after loading.
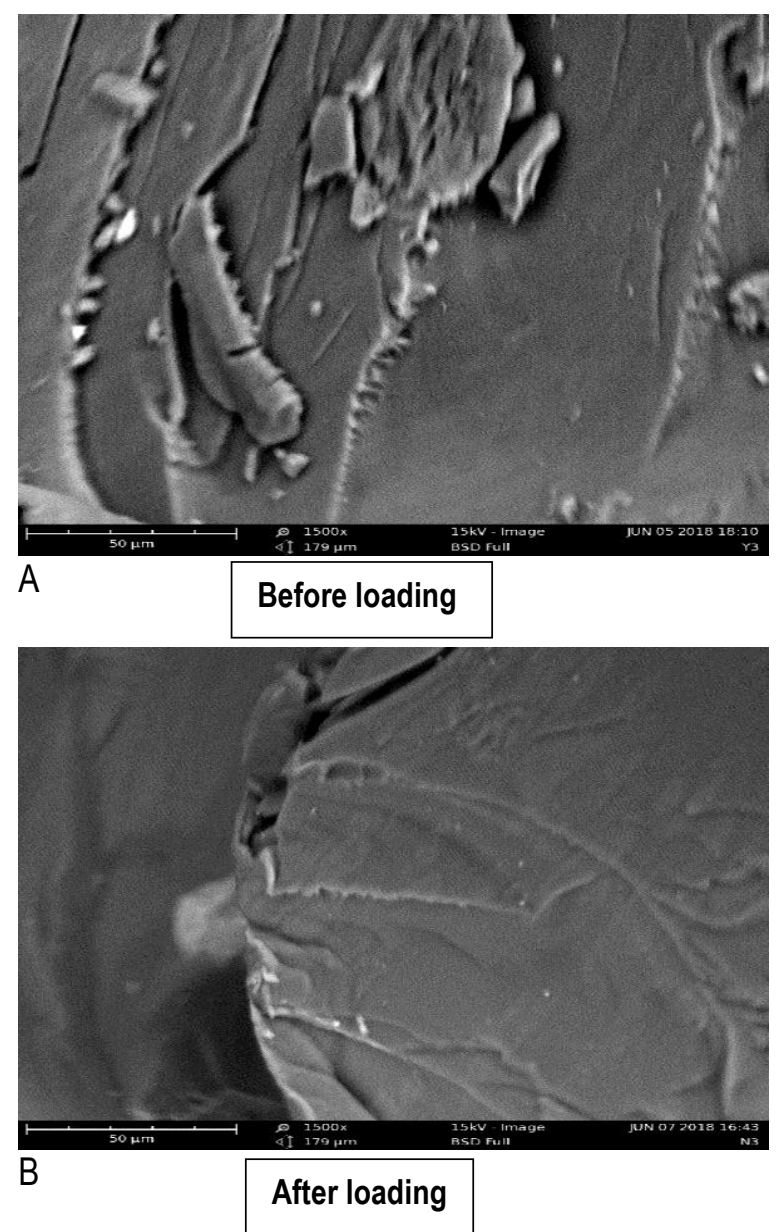

Figure 2: SEM images of S-g-Acrylamide Hydrogel Before and After Promethazine Loading

It can be seen clearly from the images that the hydrogel surface is rough and there are micropores for the accommodation of water/biological fluids and drug particles.
Figure 3 shows the percentage swelling of the prepared hydrogel in solutions of various $\mathrm{pH}$ (112).

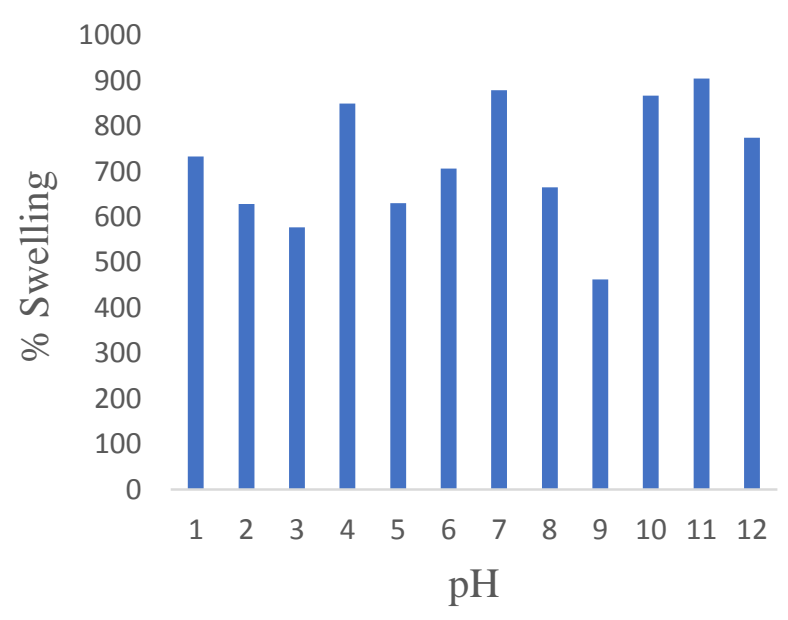

Figure 3: Percentage Swelling of Starch-gacrylamide Hydrogel.

The swelling percentage of starch-gacrylamide hydrogel suggests the hydrogel has lower absorption ability at lower $\mathrm{pH}$ when compared to the higher $\mathrm{pH}$. This is because at higher $\mathrm{pH}$ (greater than 7), there was an increase in the amount of the ionized groups which generate an electrostatic repulsion between the adjacent ions in the polymer structure of the hydrogel. Hence, this has resulted in the enhancement of water intake capacity of the hydrogel and subsequently increases the swelling capacity of the hydrogel (Mateen et al., 2016). Meanwhile at lower pHs the swelling percentage is also appreciable, this is because of the conversion of free hydroxyl groups to oxonium salt, which reduces the voids and this resulted in the low water intake by the hydrogel. Kunal et al. (2015) have previously reported similar results. Furthermore, the existence of hydrogen bond between the starch and the carbonyl groups of the acrylamide may also play a role in reducing the swelling capability of the hydrogel, because the hydrogen bond will always bring about additional cross-links to the hydrogel and this will directly reduce the swelling capability of the hydrogel. However, the decrease in swelling capacity of the hydrogel observed at higher $\mathrm{pH}$ (12) may be attributed to the fact that, at higher $\mathrm{pH}$ there is existence of sufficient sodium ions $\left(\mathrm{Na}^{+}\right)$and charge screening effect of these 
excess $\mathrm{Na}^{+}$in the swelling media. Furthermore, this may shield the carbonyl ions and prevents effective anion-anion repulsion, which would have resulted in higher swelling. This finding agrees with the work of Sadeghi, 2011.

The percentage drug release of the prepared hydrogel at various times in an enzyme-free SIF and SGF is presented in Figure 4.

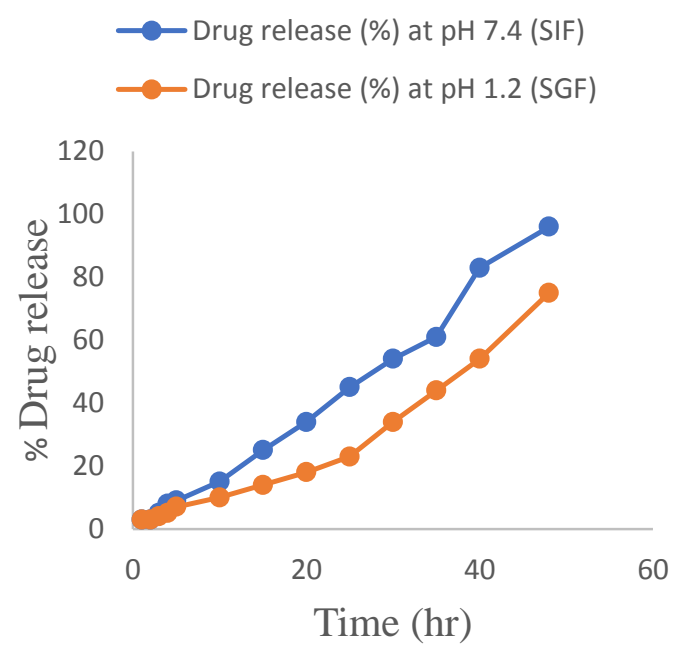

Figure 4: In vitro Release Studies of PMZ Loaded Starch-g-acrylamide in enzyme-free SGF and SIF.

After 24 hours of hydrogel swelling in the promethazine drug solution, about $99 \%$ of the drug was entrapped in the hydrogel. The results showed that during the first five hours the release was found to be $7 \%$ in SGF while it was $10 \%$ in SIF, after 10 hours the release was $24 \%$ in SIF as compared to $9 \%$ in SGF. At the end of 15 hours, the release was found to be 15 $\%$ and $27 \%$ in both SGF and SIF, respectively. The low release in SGF as compared to that of SIF can be attributed to the $\mathrm{pH}$ sensitivity of the hydrogel since the hydrogel has to swell before it releases its contents. At the end of 48 hours $89 \%$ and $96 \%$ of the drug was released in both SGF and SIF, respectively. Similar results have been reported by other researchers (Kono and Teshirogi, 2015).

\section{CONCLUSION}

In conclusion, the synthesized hydrogel was demonstrated to have excellent fluid absorption and retention capacity in solutions of various
$\mathrm{pH}$. The drug loading and release of the synthesized hydrogel have indicated that the hydrogel has excellent drug entrapment and release capacity.

\section{REFERENCES}

Ahmed E. M. (2015). Hydrogel: Preparation, Characterization and Applications. A review Journal of Advanced Research 6, 105-121.

Azman, I., Sahilah, A.M., Siti F.M.Y., Shazrul, F., Akram, N., Malina, K. and Azwan, M.L., (2015). Starch-Based Hydrogels and Their Beneficial Use as Disinfectants. Journal of Bioactive and Compatible Polymers, 22: 1-18.

Esra, A.K., Orakdogen, N., and Okay, 0. (2007). Preparation of Homogeneous Polyacrylamide Hydrogel by FreeRadical Crosslinking Copolymerization. European Polymer Journal (43): 29132921.

Jamingan, Z., Mansor, B.A., Kamaruddin, H.and Norhazlin, Z. (2015). Sago Starch Based Hydrogel Prepared Using Electron Beam Irradiation Technique for Controlled Release ApplicationMalaysian Journal of Analytical Sciences, 19: $503-512$.

Kono, H. and Teshirogi, T. (2015). Cyclodextrin-Grafted Chitosan Hydrogels for Controlled Drug Delivery. International Journal Biological Macromolecules: (72):299-308.

Kopecek, J. (2007). Hydrogel biomaterials: A Smart Future. A review Journal of Biomaterials (28):5185- 5192.

Kunal, P., Banthia, A.K., and Majumdar, D.K., (2015). Preparation of Transparent Starch Based Hydrogel Membrane with Potential Application as Wound Dressing. Journal of Biomaterial, 20(1):59-67.

Mateen, M.d., Shaikh,M., Nitin. S.G.,Wardole,A., Shrikant, V.L., (2016). Starch-Acrylic Acid Hydrogel: Synthesis, Characterization and Drug Release Study. World Journal of Pharmacy and Pharmaceutical Sciences Volume 44(07): 942-954.

Michel, B.P., Waldo, A.M., Juan, V.C.R., Alejando, M.P., Nancy, B.R., Carlos, P.C. (2011). Interpenetrated Chitosan- 
Poly (Acrylic Acid-Co-Acrylamide) Hydrogels. Synthesis, Characterization and Sustained Protein Release Studies Journal of Materials Sciences and Applications, 2: 509-512.

Muhammad, F.I., Mahmood, A. and Aysha, R. (2016). Synthesis And In Vitro Characterization of Hydroxypropyl Methylcellulose-Graft-poly (Acrylic Acid/2-Acrylamido-2 Methyl-1Propanesulfonic Acid) Polymeric Network for Controlled Release of Captopril. Acta Poloniae Pharmaceutica and Drug Research, 73(1):183-196.

Niamlang, S., Tawansorn, B., Amornrat, N. and Anuvat, S. (2013). Controlled Aloin Release from Crosslinked Polyacrylamide Hydrogels Effects of Mesh Size, Electric Field Strength and a Conductive Polymer. Journal of Materials Science, 6:4787-4800.

Park, K., Lee, SC., and Kwon, I.K., (2013). Hydrogels for Delivery of Bioactive Agents: A Historical Perspective. Journal ofAdvance Drug Delivery, 65:17-20.

Peppas, N.A., (2000). Hydrogels in pharmaceutical formulations. European
Journal of Pharmaceutics and Bio pharmaceutics, 50(1): p. 27-46.

Sadeghi M. (2011). Synthesis of Starch-g-Poly (Acrylic Acid-co-2-Hydroxy Ethyl Methacrylate) as a Potential $\mathrm{pH}$ sensitive Hydrogel-Based Drug Delivery System. Turkish Journal of Chemistry, 35: $723-733$.

Shantha, K.L. and Harding, R.K. (2002). Synthesis and Evaluation of SucroseContaining Polymeric Hydrogels for Oral Drug Delivery. Journal of Applied Polymer Science, 84: 2597-2604.

Soleimani, F. And Sadeghi, M. (2012). Synthesis of pH-Sensitive Hydrogel Based on Starch-Polyacrylate Superabsorbent Young Journal of Biomaterials and Nanobiotechnology, 3: 310-314.

Usman, M.M., Mahmood, A., Jamshed, A., Shahzeb, K. (2015).Synthesis and Characterization of Biodegradable Hydrogels for Oral Delivery Of 5 Fluorouracil Targeted to Colon. Screening with Preliminary In-Vivo Studies. Journal of Nuclear Science and Applications, 47(1): 28-40. 